\title{
Validation of pulse wave velocity as a marker of arterial stiffness
}

\author{
Laura Tripkovic ${ }^{1}$, Kathryn Hart ${ }^{1}$, Gary Frost ${ }^{2}$ and John Lodge ${ }^{1}$ \\ ${ }^{1}$ University of Surrey, Guildford, UK and ${ }^{2}$ Imperial College, London, UK
}

Arterial stiffness is an indication of structural and functional changes to the endothelium caused by factors such as aging or hypertensionrelated damage. Pulse wave velocity (PWV), a technique used to assess the extent of large artery elasticity and stiffness, has been proven to be an independent marker of CVD risk. Increases in PWV (particularly central) are linked to the presence of metabolic syndrome and a number of other associated CVD risk factors. PWV is a measure of the speed of the pulse wave as it travels down the arterial tree after the ejection of blood into the ascending aorta. The higher the velocity of the wave, the less elastic the arterial walls, thus indicating increased arterial stiffness. This technique is simple and non-invasive, with reportedly good levels of reproducibility. The aim of the present study is to determine intra and inter-individual variation and establish predictive markers of PWV within a heterogenous population group to inform its use in research and clinical practice.

PWV was performed on twenty-two males aged 21-56 years. All participants were normotensive, with a BMI range of 18.7-33.5 kg/ $\mathrm{m}^{2}$. All participants consumed a standard meal on the evening before the measurements session and then fasted for $12 \mathrm{~h}$. Following a standardised operating procedure PWV measurements (peripheral (p) and central (c)) were recorded in duplicate within one session for each participant.

Group ( $n$ 23) intra-individual variations for pPWV and cPWV, expressed as CV, were found to be $8.4 \%$ and $5.9 \%$ respectively. Only age was predictive for pPWV, accounting for $32 \%$ of the variance $(P=0.002)$, whilst a model incorporating age and diastolic and systolic blood pressure accounted for $72 \%$ of the variance in cPWV $(P<0.001)$. Younger males $(21-30$ years) had significantly lower pPWV (m/s; 7.0 (SD 1.31); $P>0.05$ ) and cPWV (5.7 (SD 0.74); $P>0.001)$ than the older males (31-56 years; pPWV 8.8 (SD 1.14$) \mathrm{m} / \mathrm{s} ; \mathrm{cPWV} 8.5$ (sD $2.19) \mathrm{m} / \mathrm{s})$. There was a significant correlation between cPWV and SBP $(P=0.02$; Figure).

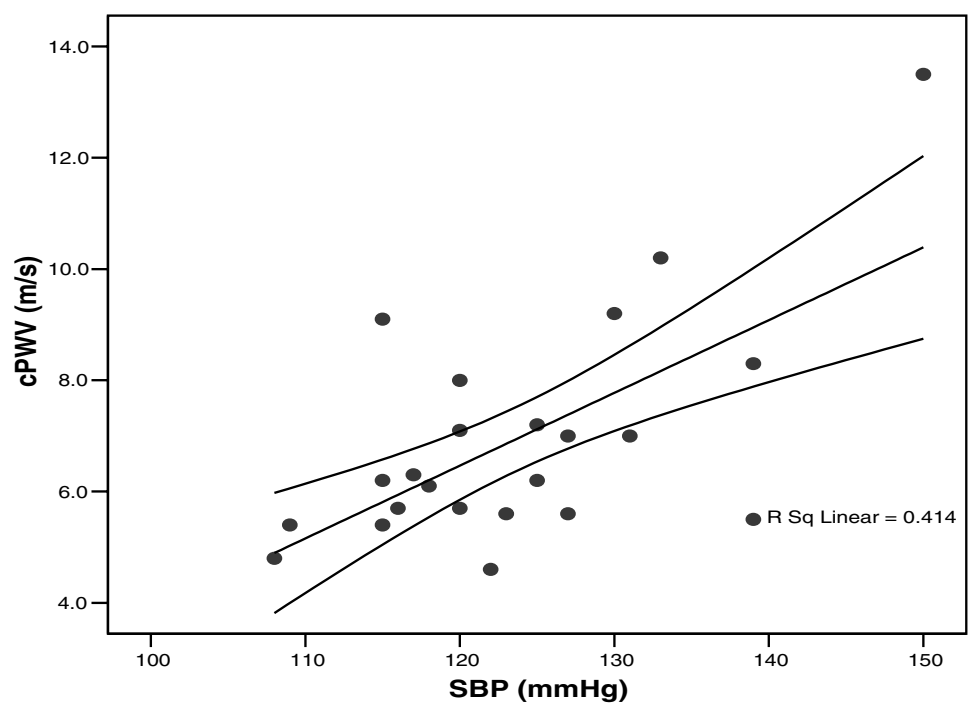

Figure. A strong predictive relationship between systolic blood pressure (SBP) and cPWV ( $n$ 23, $r 0.475, P=0.02)$.

The results show relatively low intra-individual variation and an apparent relationship between blood pressure and cPWV, which supports current evidence ${ }^{(1)}$. There were strong positive relationships between cPWV and known CVD risk factors; further indication that PWV is a valid marker for CVD. The technique has proven to be robust and highly reproducible, and is now being extensively used within a variety of nutrition intervention studies to further investigate relationships with other validated markers of CVD risk. Ongoing research will also evaluate possible limiting factors that may affect PWV in order to establish its optimum use within the classical research setting.

1. Schillaci G, Pirro M, Vaudo G, Mannarino MR, Savarese G, Pucci G, Franklin SS \& Mannarino E (2005) Hypertension 45, 1078-1082. 